\title{
gs \\ Importance of intramolecular electron spin relaxation in small molecule semiconductors
}

\author{
L. Schulz, ${ }^{1}$ M. Willis, ${ }^{2}$ L. Nuccio, ${ }^{2}$ P. Shusharov,${ }^{2}$ S. Fratini, ${ }^{3}$ F. L. Pratt ${ }^{4}$ W. P. Gillin, ${ }^{2}$ T. Kreouzis, ${ }^{2}$ M. Heeney, ${ }^{5}$ \\ N. Stingelin, ${ }^{5}$ C. A. Stafford, ${ }^{6}$ D. J. Beesley, ${ }^{5}$ C. Bernhard, ${ }^{1}$ J. E. Anthony, ${ }^{7}$ I. McKenzie, ${ }^{4}$ J. S. Lord, ${ }^{4}$ and A. J. Drew ${ }^{1,2}$ \\ ${ }^{1}$ Department of Physics and Fribourg Center of Nanomaterials, University of Fribourg, CH-1700 Fribourg, Switzerland \\ ${ }^{2}$ Queen Mary University of London, Department of Physics, London, E1 4NS, United Kingdom \\ ${ }^{3}$ Institut Néel CNRS, F-38042 Grenoble, France \\ ${ }^{4}$ ISIS Muon Facility, Rutherford Appleton Laboratory, Didcot, OX11 0QX, United Kingdom \\ ${ }^{5}$ Centre for Plastic Electronics, Imperial College London, London, SW7 2AZ, United Kingdom \\ ${ }^{6}$ Department of Physics, University of Arizona, Tucson, Arizona, USA \\ ${ }^{7}$ Department of Chemistry, University of Kentucky, Lexington, Kentucky 40506, USA
}

(Received 13 April 2011; published 26 August 2011)

\begin{abstract}
Electron spin relaxation rate (eSR) is investigated on several organic semiconductors of different morphologies and molecular structures, using avoided level crossing muon spectroscopy as a local spin probe. We find that two functionalized acenes (polycrystalline tri(isopropyl)silyl-pentacene and amorphous 5,6,11,12tetraphenyltetracene) exhibit eSRs with an Arrhenius-like temperature dependence, each with two characteristic energy scales similar to those expected from vibrations. Polycrystalline tris(8-hydroxyquinolate)gallium shows a similar behavior. The observed eSR for these molecules is no greater than $0.85 \mathrm{MHz}$ at $300 \mathrm{~K}$. The variety of crystal structures and transport regimes that these molecules possess, as well as the local nature of the probe, strongly suggest an intramolecular phenomenon general to many organic semiconductors, in contrast to the commonly assumed spin relaxation models based on intermolecular charge-carrier transport.
\end{abstract}

DOI: 10.1103/PhysRevB.84.085209

PACS number(s): 75.76.+j, 75.47.Pq, 76.75.+i, 81.05.Fb

\section{INTRODUCTION}

Research into organic semiconductors has progressed at a phenomenal rate over the last few decades, with great success in moving from the initial stages of understanding the fundamental physics, through to today where products are entering the market. ${ }^{1}$ Besides their ability to transport charge or emit light, there has recently been an interest in organic semiconductors as candidates for future spin-based technologies. This is thanks to their long spin coherence time and their suitability as a system for studying the fundamental spin phenomena relevant to many materials and applications. $^{2-5}$

Traditional explanations for their long spin coherence time $\tau_{e}$ are a weak hyperfine coupling of the electron's spin to the nuclear spins ${ }^{6}$ or a weak spin-orbit (SO) interaction due to organic semiconductors being composed of light species. ${ }^{7}$ Currently, it is not clear what the dominant spin relaxation mechanism is, as there is a lack of experimental techniques that can directly probe $\tau_{e}$ microscopically.

Current experimental estimates of $\tau_{e}$ vary between a few microseconds to a second or more, ${ }^{5,8}$ which may be representative of the indirect nature of the experimental measurement. In these measurements, spin coherence times were extracted from magnetotransport measurements on unipolar spin-valve-like structures. $^{3,6,8}$ This approach requires a robust theoretical model; those applied thus far are analogs of the Elliot-Yafet ${ }^{9}$ or D'yakonov-Perel mechanisms, ${ }^{10}$ or one involving electrons observing a random hyperfine field upon hopping between molecular sites. ${ }^{11}$ All of these theoretical models are transport based and require an accurate measure of the charge-carrier mobility, whose uncertainty ${ }^{2,12}$ even in well-defined organic light-emitting diode (OLED) structures leads to a significant error in the value of $\tau_{e}$. Electron paramagnetic resonance (EPR) is potentially capable of providing this information, but it requires a material with intrinsic electrons or electrons provided by doping, which is not always possible or desirable.

In the following, we show that muon spin relaxation/resonance $(\mu \mathrm{SR})$ can be used to measure the intrinsic electron spin relaxation rate and we find strong evidence for an intramolecular based spin relaxation mechanism in several different molecules with an acene backbone. Furthermore, from measurements on a different molecule based on hydroxyquinolate, it appears that this mechanism may be quite general.

\section{SAMPLES AND EXPERIMENTAL METHOD}

\section{A. Sample preparation and properties}

We performed avoided level crossing (ALC) measurements on two functionalized acenes 6,13-bis(tri(isopropyl) silylethynyl) pentacene (TIPS-pentacene) and 5,6,11,12tetraphenyltetracene (rubrene), based on a pentacene and tetracene backbone, and tris(8-hydroxyquinolate)gallium $\left(\mathrm{Gaq}_{3}\right)$ with a tri-ligand molecular structure. Their molecular structure is shown in Fig. 1. TIPS-pentacene was synthesized according to a published procedure, ${ }^{13,14}$ and was additionally purified by repeated recrystallization from dichloromethane/ethanol. The measured TIPS-pentacene sample was a polycrystalline powder. Sublimed grade rubrene was purchased from Aldrich. Amorphous rubrene was then prepared by first heating the powder to above its melting temperature $\left(355^{\circ} \mathrm{C}\right)$ between two glass slides to prevent sublimation of the material, with subsequent quenching on metal plates kept at $0{ }^{\circ} \mathrm{C}$. $\mathrm{Gaq}_{3}$ was synthesized using a published method ${ }^{15}$ and purified using train sublimation, resulting in a polycrystalline structure. These materials were chosen due to the pronounced differences in solid-state packing. TIPS-pentacene crystallizes in a two-dimensional brickwork 


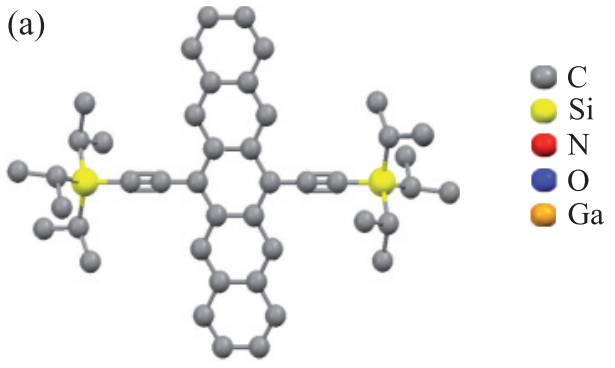

(b)

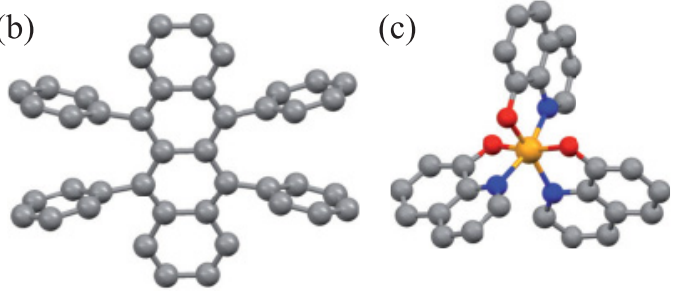

FIG. 1. (Color online) Molecular structures of (a) TIPSpentacene, (b) rubrene, and (c) $\mathrm{Gaq}_{3}$ (Refs. 13, 14, and 22-24). Hydrogen has been omitted for clarity.

arrangement, with close $\pi-\pi$ overlap, which results in very high charge-carrier mobilities. ${ }^{16,17}$ In contrast, the rubrene investigated here was an amorphous powder and as a consequence had very poor charge transport. $\mathrm{Gaq}_{3}$, an analog of tris(8-hydroxyquinolate)aluminium $\left(\mathrm{Alq}_{3}\right)$, crystallizes with a one-dimensional helical packing. ${ }^{18}$

\section{B. Muon spin relaxation technique}

The $\mu$ SR technique is based on positively charged surface muons with near $100 \%$ spin polarization, which are implanted into the organic samples. The muons can either thermalize as diamagnetic positively charged species or form a hydrogenlike muonium. ${ }^{18-21}$ Due to unsaturated bonds that are present in the molecules, this muonium can react with a molecule creating a paramagnetic molecule. In a "longitudinal field" experiment a magnetic field is applied parallel to the muon's initial spin direction. The energy levels of the singlet/triplet states of the bound muonium are tuned via the Zeeman interaction. ${ }^{21}$ In high magnetic fields, where most of these experiments were carried out, the eigenstates of the spin systems are to a good approximation pure Zeeman product states. Muons implanted with their spins parallel or antiparallel to the field are thus in an eigenstate, and no time evolution of spin polarization is expected. ${ }^{25}$ At a particular longitudinal field cross relaxation effects produce a so-called avoided level crossing (ALC), at what would otherwise be energy-level degeneracies, and eigenstates are mixtures between two Zeeman states. Whenever the two mixing levels belong to different muon magnetic quantum states, the muon's spin polarization oscillates between the two. The position and linewidth of these so-called ALC resonances are determined by the muon-electron isotropic and anisotropic hyperfine coupling (HFC). ${ }^{21,25}$

In solids, dipolar coupling drives a single-particle muon spin-flip $\left(\Delta_{1}\right)$ transition, which is absent in liquids and is independent of the other nuclei in the system. The width of the $\Delta_{1}$ line depends on the dipolar coupling strength but its intensity (asymmetry change) remains constant. A muon-nuclear-spin flip-flop $\left(\Delta_{0}\right)$ transition can also sometimes be observed in solids but is generally weaker than the $\Delta_{1}$. Importantly, it broadens with increasing dipolar coupling and the intensity at any one field drops, resulting in a relatively small contribution to the experimental data (as demonstrated and discussed in the Appendix). The muon-nuclear-spin flip-flip transition $\left(\Delta_{2}\right)$ is also negligibly small, since the coupling frequencies are small. In solids therefore experimental data tend to be dominated by the $\Delta_{1}$ transitions, which is an extraordinarily sensitive probe of radical dynamics ${ }^{25-27}$ including the electron spin relaxation rate. ${ }^{25,28}$

The muon experiments were performed on the ARGUS, EMU, and HIFI spectrometers at the ISIS Muon Source, Rutherford Appleton Laboratory and the DOLLY spectrometer at the Laboratory for Muon Spectroscopy, Paul Scherrer Institute. $115 \mathrm{mg}$ of TIPS-pentacene, $190 \mathrm{mg}$ of rubrene, and $800 \mathrm{mg}$ of $\mathrm{Gaq}_{3}$ were placed into $15 \times 25 \mathrm{~mm}$ envelopes made from $25 \mu \mathrm{m}$ thick silver foil (99.99\% pure). To ensure the muons stopped in the organic material, one further layer of the $25 \mu \mathrm{m}$ silver foil was placed on top to act as a degrader. For the experiments performed at ISIS, "fly-past" mode was used such that $\sim 95 \%$ of the measured muons were implanted in the sample with the remaining $\sim 5 \%$ stopping in the silver foil or sample holder. All remaining muons were able to exit into a long tube and stop at the end, where their decay positrons were out of range of the detectors. Active veto was not necessary at PSI, as the beam area was smaller than the well-centered sample.

\section{RESULTS AND DISCUSSION}

\section{A. Data analysis: ALC simulation}

Figure 2(a) shows the time-integrated data ${ }^{21,29}$ measured for TIPS-pentacene at $300 \mathrm{~K}$. Data analysis was made using the program WimDA. $^{30}$ The shown curve is the so-called repolarization curve representing the realignment of the muon spin ensemble in the external magnetic field. It can be also seen that an ALC line shape appears at about $0.3 \mathrm{~T}$ as indicated by the black arrow. Because the ALC line shape is placed on the slope of the repolarization curve, the ALC has to be extracted from the repolarization curve. To this end, the repolarization data were fitted to a four-component repolarization function. ${ }^{21,29}$ The points within the ALC were omitted from the fit, which is shown as a solid line. As the slope region of the repolarization curve was temperature independent, the $300 \mathrm{~K}$ fit could be also used for other temperatures as indicated in the inset of Fig. 2(a). The extracted ALC line shape for the case of $10 \mathrm{~K}$ can be seen in Fig. 2(b). For rubrene, we used a two-component anisotropic repolarization curve with a superimposed ALC. And for the ALC measured on $\mathrm{Gaq}_{3}$, we used a linear background using points outside the range of the ALC, since the ALC is located at much higher magnetic fields well away from any repolarization.

The extracted ALC for TIPS-pentacene at $10 \mathrm{~K}$, shown in Fig. 2(b), is close to $0.3 \mathrm{~T}$ corresponding to a low isotropic HFC constant of approximately $80 \mathrm{MHz}$. The shape of the ALC suggests an involvement of two very close individual $\Delta_{1}$ transitions. They correspond to the muonium addition sites at the spine and the triple bond of the side group. The muonium states and sites are further discussed with the help 

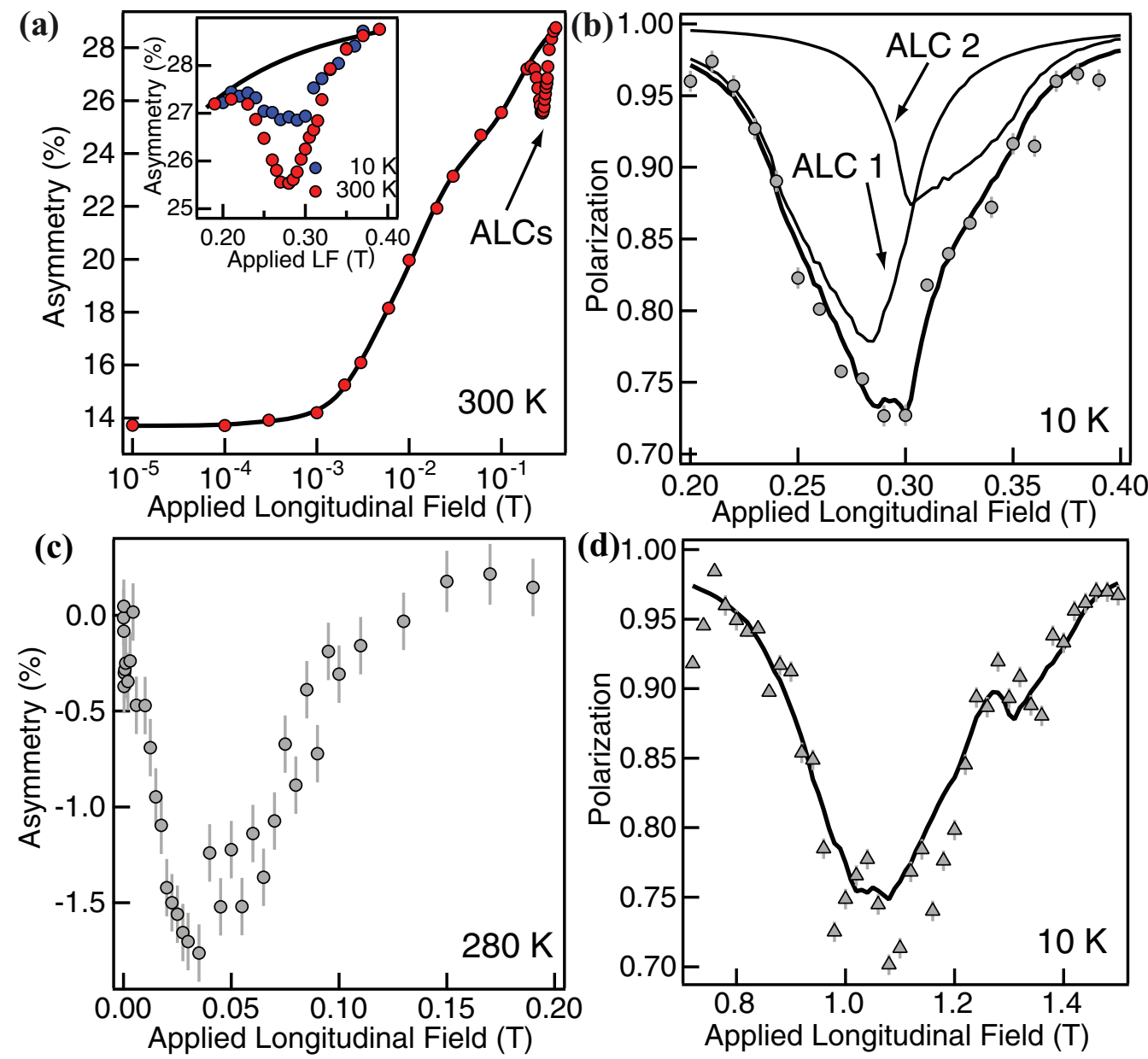

FIG. 2. (Color online) (a) The repolarization curve for TIPS-pentacene at $300 \mathrm{~K}$ was fitted to a repolarization function (solid line, see text) in order to extract the avoided level crossings (ALCs) at about $0.3 \mathrm{~T}$. The fitted function could be used for all temperatures (see inset). (b) The extracted ALC for TIPS-pentacene at $10 \mathrm{~K}$ is in fact composed of two individual ALCs. The simulated ALC (solid line) exhibits good agreement with the data. (c) The extracted ALC for rubrene at $280 \mathrm{~K}$. In this case, a simulation of the ALC was unfortunately impossible due to its high relative width (see text). (d) $\mathrm{For}_{\mathrm{Gaq}}$, the simulations again represent the data well.

of high transverse field measurements and DFT calculations in the Appendix. We have thus simulated the TIPS data as two independent $\Delta_{1}$ transitions, assuming an electron spin relaxation rate of $0.02 \mathrm{MHz}$ at low temperatures (the lower limit of our sensitivity given our statistics). This assumption is justified if the electron spin relaxation rate is strongly governed by a temperature-dependent effect, such as if it were coupled to the population of vibrational modes. The simulations of the two $\Delta_{1}$ ALCs have been done with the software QUANTUM. ${ }^{31}$ The dynamical calculation with a Monte Carlo (MC) powder averaging was used, with about $10000 \mathrm{MC}$ steps for each magnetic field. The obtained values for the isotropic and anisotropic HFCs, as well as the relative amplitudes of the two ALCs, are shown in Table I. The simulated ALC line shape (solid line) exhibits good agreement with the data.

The analysis of the rubrene spectra [280 K shown in Fig. 2(c)] has proven to be more difficult, as unfortunately no reliable simulation of the ALC could be carried out. We were unable to establish the scaling factor between polarization and asymmetry, related to the width and strength of the ALC, which is in turn related to a range of muon-electron hyperfine coupling constants. Crystallographic disorder present in the amorphous rubrene results in the distribution of hyperfine coupling constants observed in our measurements.

For the $\mathrm{Gaq}_{3}$, the simulated ALCs were obtained by applying a similar approach to TIPS-pentacene-assuming all peaks present are due to $\Delta_{1}$ transitions. Although we started with the $10 \mathrm{~K}$ data set first [Fig. 2(d)], we took into account that at $300 \mathrm{~K}$ there are three individual ALCs (see Sec. III B).

TABLE I. Simulated parameters for the $10 \mathrm{~K}$ TIPS-pentacene. The value for the eSR at $10 \mathrm{~K}$ represents our sensitivity limit. All values are in $\mathrm{MHz}$ (except for the rel. amplitude). $A, D$, and $E$ define the anisotropic hyperfine tensor (Ref. 31 ), whose principal values are $(A+D, A-D / 2+E / 2, A-D / 2-E / 2)$. These parameters correspond to two components centered on 0.28 and $0.31 \mathrm{~T}$.

\begin{tabular}{lcc}
\hline \hline & ALC 1 $(\mathrm{MHz})$ & ALC 2 $(\mathrm{MHz})$ \\
\hline$A$ & $75.0 \pm 0.8$ & $86.4 \pm 0.9$ \\
$D$ & $-9.0 \pm 0.7$ & $9.0 \pm 0.7$ \\
$E$ & $5.0 \pm 1.1$ & $1.0 \pm 0.2$ \\
eSR & 0.02 & 0.02 \\
Rel. amplitude & 0.64 & 0.36 \\
\hline \hline
\end{tabular}


TABLE II. Simulated parameters for the $10 \mathrm{~K} \mathrm{Gaq}_{3}$. The value for the eSR at $10 \mathrm{~K}$ represents our sensitivity limit. All values are in $\mathrm{MHz}$ except for the relative amplitudes. $A, D$, and $E$ define the anisotropic hyperfine tensor (Ref. 31), whose principal values are $(A+D, A-$ $D / 2+E / 2, A-D / 2-E / 2)$. These parameters correspond to three components centered on $1,1.1$, and $1.35 \mathrm{~T}$.

\begin{tabular}{lccc}
\hline \hline & ALC 1 $(\mathrm{MHz})$ & ALC 2 $(\mathrm{MHz})$ & ALC 3 (MHz) \\
\hline A & $265 \pm 1$ & $295 \pm 1$ & $365 \pm 1$ \\
D & $-30.0 \pm 21$ & $37 \pm 4$ & $20 \pm 5$ \\
E & $0 \pm 21$ & $37 \pm 2$ & $15 \pm 6$ \\
eSR & 0.02 & 0.02 & 0.02 \\
Rel. amplitude & 0.15 & 0.65 & 0.20 \\
\hline \hline
\end{tabular}

The isotropic HFC constants (see Table II) are close to values reported for other aromatic systems elsewhere. ${ }^{32-34}$

\section{B. Electron spin relaxation rate}

Figure 3(a) shows the ALC measured for TIPS-pentacene at 10 and $300 \mathrm{~K}$. The one common and most prevalent feature in these spectra is the clear and unambiguous increase of the ALC size at high temperature. From the time-differential data at 0.27 T, shown in Fig. 3(b), it is clear that this increased ALC depth is related to a higher relaxation rate of the muon spins. The time-dependent data have been fitted to an exponential function. The characteristic relaxation rate $\lambda$ is plotted as a function of the magnetic field in Fig. 3(c). It begins to increase away from the expected power-law behavior [shown by the lines in Fig. 3(c)], with a pronounced peak centered on the location of the ALC. The observed data discount any nonrelaxing phenomena, such as changes in muonium formation probability or background.

One possible explanation for the data shown in Fig. 3 could be a time-dependent modulation of the spin density sampled by the muon, as a result of periodic structural changes, such as phonons or intramolecular vibrations. However, as is clear from the temperature-independent width and position of the ALC, the isotropic and anisotropic HFCs are not particularly temperature dependent. One would expect a much larger temperature dependence of the HFCs, if this modulation was responsible. Any rotational molecular dynamics, such as those observed in C60, can be discounted since our sample was solid without a rotational degree of freedom and there is no change in shape and/or position of the ALC, as would be expected. ${ }^{32}$ Finally, although surrounding nuclear spins can have an effect on the shape of the ALC, they cannot account for the dramatic increase in relaxation rate, because of their small coupling strength to the muon spin. In the Appendix, it is demonstrated that the impact of a proton spin on the $\Delta_{1}$ resonance is negligible compared to the measured effect.

The only relaxation phenomenon that can reasonably describe the data shown in Fig. 3 is an increase in the radical eSR, such that it is within the muon's experimental time window. For intermediate eSRs $(0.01<\mathrm{eSR}<\sim 1 \mathrm{MHz})$, neither the position nor the absolute width of the ALC are significantly affected. The main effect of this additional electron spin relaxation is a change in ALC intensity, as a result of an increased time-dependent relaxation, ${ }^{25,28}$ which is
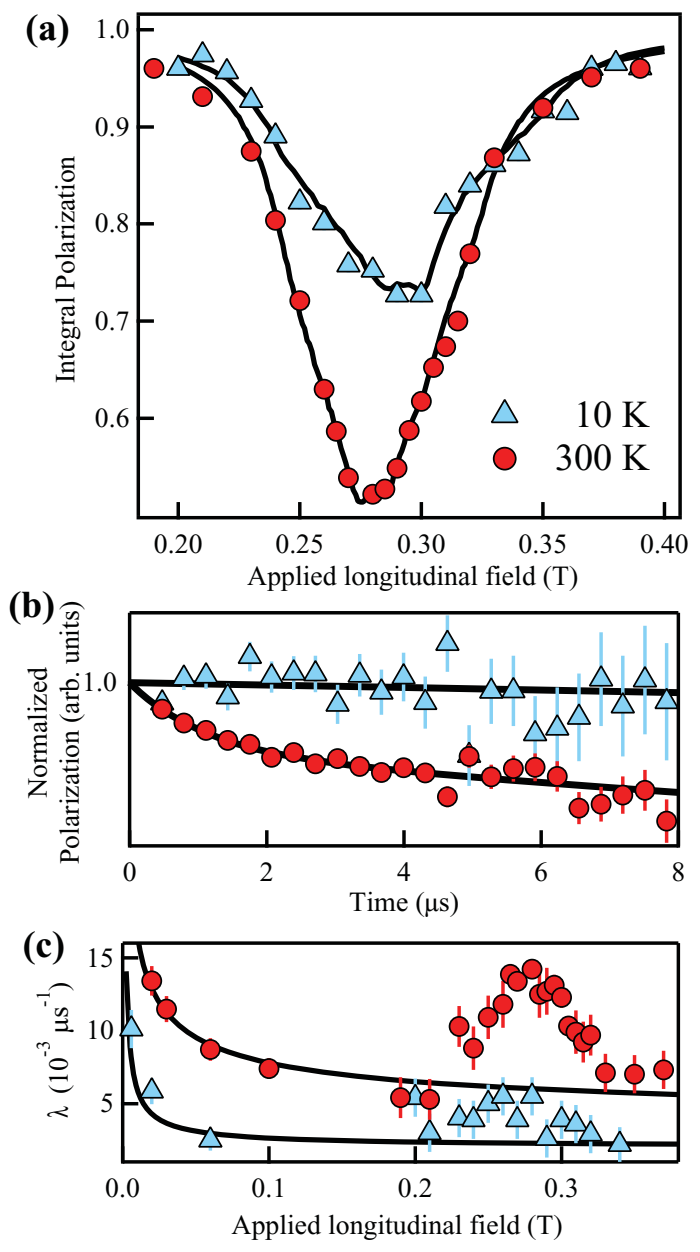

FIG. 3. (Color online) Muon data for TIPS-pentacene for $T=$ $300 \mathrm{~K}$ (red circles) and $T=10 \mathrm{~K}$ (blue triangles). (a) The muon spin polarization around the avoided level crossings (ALC). Modeling for these ALCs is indicated by the black lines (see text). (b) The time-dependent muon polarization at $0.27 \mathrm{~T}$. Data are plotted using a linear scale and the black lines correspond to fits to an exponential function, from which the relaxation rate shown in (c) is extracted. (c) The fitted field-dependent exponential muon spin relaxation rate $\lambda$ showing that there is a peak in the muon spin relaxation rate around the position of the ALCs. The lines represent the expected power-law dependence for the off-resonant relaxation rate.

exactly as observed in the data shown in Fig. 3(a). Using a spin density matrix formalism ${ }^{23,31}$ to model our spectra [solid lines in Fig. 3(a)], we extracted an eSR of $0.76 \pm 0.03 \mathrm{MHz}$ at 300 $\mathrm{K}$, assuming an eSR of $0.02 \pm 0.01 \mathrm{MHz}$ at $10 \mathrm{~K}$ (the lower limit of our sensitivity with reasonable statistics). The whole set of simulation parameters is listed in Table III.

Figure 4 shows the eSR for TIPS-pentacene plotted on a logarithmic scale against inverse temperature. On this Arrhenius plot, the eSR reduces linearly with two characteristic energy scales of $19 \pm 2 \mathrm{meV}$ and $3.2 \pm 0.2 \mathrm{meV}$, which are a similar energy to the molecular vibrations common in organic materials, suggesting that there is a coupling of eSR to the population of these vibrations via a direct spin-vibration coupling active at the molecular level.

As discussed earlier, many of the theoretical models applied to explain eSR are transport based and as such, an Arrhenius 
TABLE III. Simulated parameters for TIPS-pentacene at $300 \mathrm{~K}$. All values except the relative amplitudes are in $\mathrm{MHz}$. These parameters correspond to two components centered on 0.27 and $0.3 \mathrm{~T}$.

\begin{tabular}{lcc}
\hline \hline & ALC 1 $(\mathrm{MHz})$ & ALC 2 $(\mathrm{MHz})$ \\
\hline$A$ & $73.3 \pm 1.0$ & $82.6 \pm 1.2$ \\
$D$ & $-6.3 \pm 0.5$ & $5.5 \pm 0.5$ \\
$E$ & $4.0 \pm 0.3$ & $4.0 \pm 0.3$ \\
eSR & $0.76 \pm 0.03$ & $0.76 \pm 0.03$ \\
Rel. amplitude & 0.64 & 0.36 \\
\hline
\end{tabular}

behavior to eSR is not necessarily surprising. One would therefore expect the spin relaxation rate to be significantly determined by the mobility. In our case, where we measure an electron localized to a single molecule, one needs to discount a relaxation phenomenon resulting from any other free charges, present due to track electrons resulting from the muon's thermalization or doping from impurities. We show below that this is not the case and there is indeed a localized relaxation mechanism responsible for eSR. We compare the results from amorphous rubrene-a tetracene derivative [see Fig. 1(b)] —with those from TIPS-pentacene. From Figs. 5(a)-5(c) it is immediately clear that there is a broad and intense ALC resonance at a low magnetic field, which is associated with a time differential relaxation. Furthermore, both the ALC amplitude and muon spin relaxation rate show a large magnitude change with temperature. In common with the TIPS-pentacene, the temperature dependence of eSR in rubrene shown in Fig. 6 also has an Arrhenius nature, with two characteristic energy scales of $0.52 \pm 0.04 \mathrm{meV}$ and $1.15 \pm 0.02 \mathrm{meV}$. Unfortunately it is not possible to model the rubrene data exactly as we have in TIPS-pentacene, since the local disorder results in an unquantifiable distribution of muon-

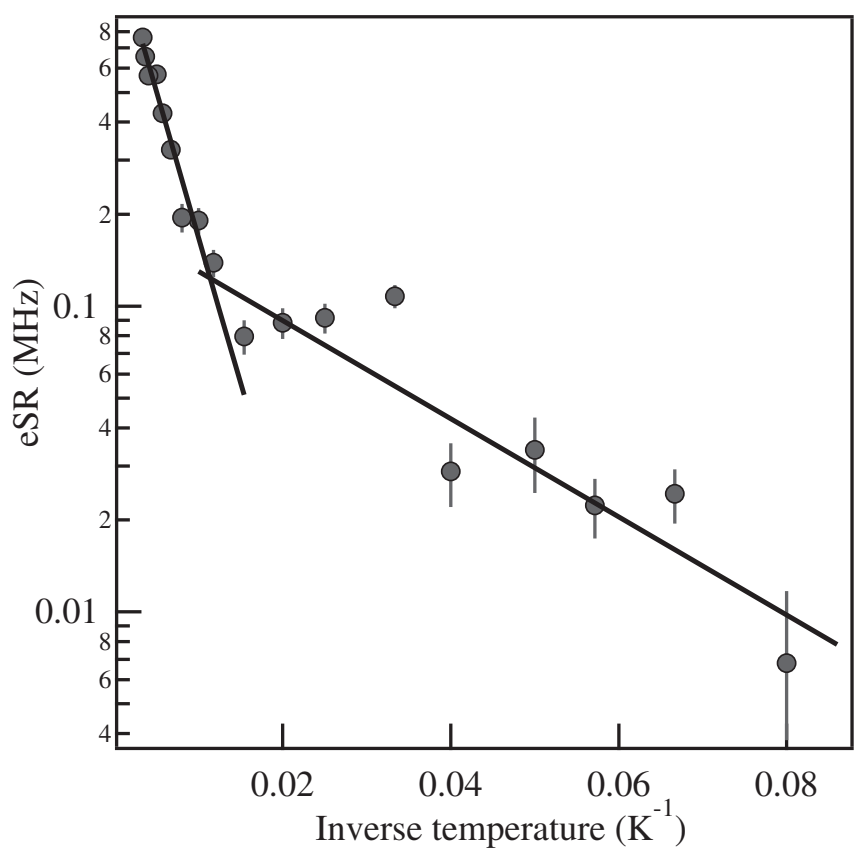

FIG. 4. The electron spin relaxation rate for TIPS-pentacene on an Arrhenius plot with two characteristic energy scales (see text).
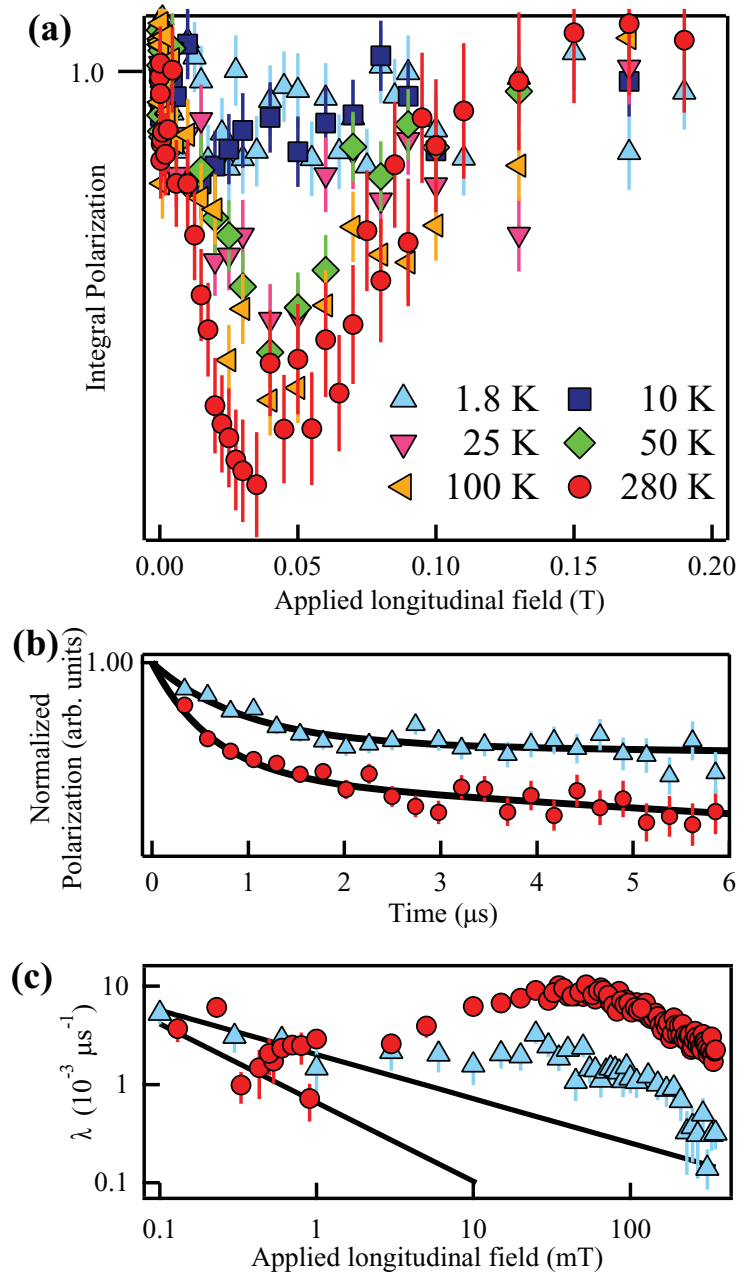

FIG. 5. (Color online) (a) Plot of the muons' field-dependent spin polarization for rubrene, showing there is a broad and intense ALC. (b) The time-dependent muon spin polarization at $0.03 \mathrm{~T}$ for $T=$ $1.8 \mathrm{~K}$ and $T=280 \mathrm{~K}$, plotted using a linear scale. (c) The fitted fielddependent exponential relaxation rate $\lambda$. The lines show the expected power-law dependency of the off-resonant background relaxation at high fields.

electron HFCs as discussed in Sec. III A. The temperaturedependent eSR shown in Fig. 6 is therefore in arbitrary units, but the trend is clearly the same as in TIPS-pentacene.

The similarity between the TIPS-pentacene and rubrene data is quite surprising. As discussed earlier, the crystal structures of the materials are quite different, resulting in a high charge-carrier mobility along two axes for TIPS-pentacene and no axes for amorphous rubrene. Given the similarities in results on these materials, it is clear that electron spin relaxation is not simply a transport phenomenon as is often assumed for organic materials. ${ }^{3,6-10,35}$ In addition to any transport-based spin relaxation that may be present, there is an intramolecular process giving spin relaxation. Interestingly, Arrhenius eSR is also observed in tetrathiafulvalene (TTF), ${ }^{36}$ opening up the possibility that this local relaxation mechanism is more generally applicable across the range of molecular semiconductors. To assess the possibility of generality further, we performed high-field ALC measurements on $\mathrm{Gaq}_{3}$, which has a triligand molecular structure and does not contain 


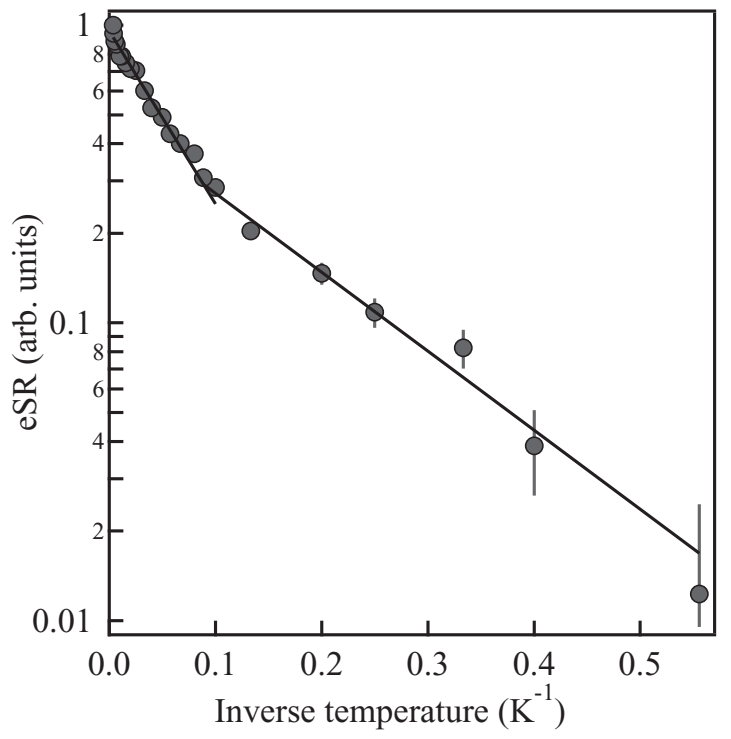

FIG. 6. The temperature dependence of the electron spin relaxation for rubrene shows Arrhenius behavior also with two characteristic energy scales (see text).

an acene backbone [see Fig. 1(c)]. In Fig. 7(a) we plot time-integrated muon spin polarization data for 300 and $3 \mathrm{~K}$, where a series of ALC resonances is found between 0.8 and $1.5 \mathrm{~T}$, around which an elevated muon spin relaxation rate is observed. Most importantly, the muon spin relaxation rate in the region of the ALC and its amplitude are significantly larger at $300 \mathrm{~K}$ compared to $3 \mathrm{~K}$, with little or no change to position or shape. This is very similar to all the previously presented data. Modeling in a similar manner to the other molecules, we find that the eSR rises from $0.02 \pm 0.01 \mathrm{MHz}$ at $3 \mathrm{~K}$ to $0.85 \pm 0.02 \mathrm{MHz}$ at $300 \mathrm{~K}$. The obtained parameters are listed in Table IV.

\section{Discussion}

Whilst we do not have sufficient data to show an Arrhenius temperature dependence for $\mathrm{Gaq}_{3}$, nonetheless the similarity suggests that it is the same mechanism responsible for the eSR in all of the molecules presented here, in addition to TTF. ${ }^{36}$
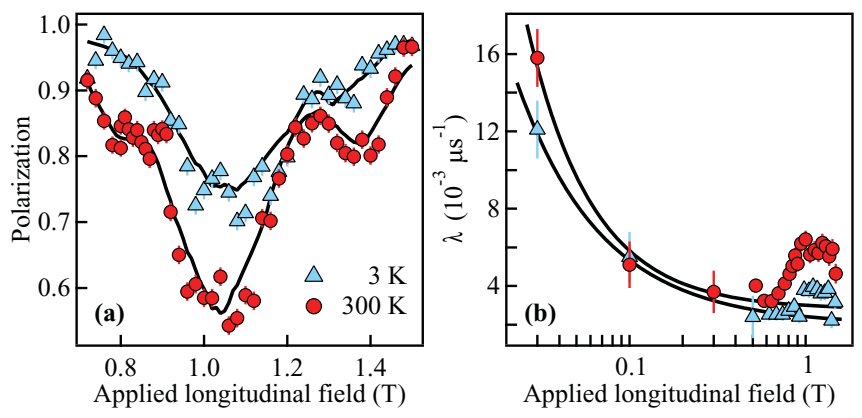

FIG. 7. (Color online) (a) A series of ALCs in polycrystalline $\mathrm{Gaq}_{3}$ around $1 \mathrm{~T}$, for $300 \mathrm{~K}$ (red circles) and $3 \mathrm{~K}$ (blue triangles). (b) An increase in relaxation rate $\lambda$ is observed in the region where the ALCs are observed. The temperature dependence of both (a) and (b) are very similar to the acene molecules, suggesting a common mechanism.
TABLE IV. Simulated parameters for the $300 \mathrm{~K} \mathrm{Gaq}_{3}$. All values except the relative amplitudes are in $\mathrm{MHz}$. These parameters correspond to three components centered on $0.78,1.04$, and $1.36 \mathrm{~T}$.

\begin{tabular}{lccc}
\hline & ALC 1 $(\mathrm{MHz})$ & ALC 2 $(\mathrm{MHz})$ & ALC 3 $(\mathrm{MHz})$ \\
\hline$A$ & $215 \pm 1$ & $282 \pm 1$ & $375 \pm 1$ \\
$D$ & $-15 \pm 10$ & $30 \pm 3$ & $17 \pm 5$ \\
$E$ & $-15 \pm 6$ & $30 \pm 2$ & $17 \pm 6$ \\
eSR & $0.85 \pm 0.02$ & $0.85 \pm 0.02$ & $0.85 \pm 0.02$ \\
Rel. amplitude & 0.15 & 0.65 & 0.20 \\
\hline \hline
\end{tabular}

We therefore suggest that the coupling of eSR to molecular vibrations could be a mechanism general to all small molecular systems.

Interestingly, at the fields presented here (up to $1.5 \mathrm{~T}$ ) the nuclear spins are effectively decoupled from the electron and muon spins. ${ }^{21}$ Models based on the HF interaction, such as the one proposed by Bobbert and co-workers ${ }^{11}$ or an equivalent localized mechanism, cannot therefore account for the relaxation effects measured here. Another possible spin relaxation mechanism would be the spin-orbit interaction, which is consistent with the small effect of deuteration on the intrinsic organic magnetoresistance. ${ }^{37}$ Previously, an Elliot-Yafet-like spin-orbit mechanism has been suggested as a possible driver of spin relaxation, ${ }^{5,6}$ where the eSR is inversely proportional to the charge-carrier mobility. However, this and many other spin-orbit based mechanisms is incompatible with the experimental data presented here, which show that the mechanism must be a localized one. Another possible relaxation mechanism is spin flips induced by the exchange interaction with passing conduction electrons or holes. These charge carriers need not have any other spin relaxation mechanisms of their own and could easily have a long spin relaxation time while giving a radical-electron relaxation, since there may be more free carriers than bound radicals at $300 \mathrm{~K}$, even in a nominally pure and undoped semiconductor as measured here. However, we have already experimentally shown that such a mechanism is unlikely, since a strong temperature-dependent eSR is present in the two-dimensional brickwork arrangement of TIPS-pentacene, which results in very high charge-carrier mobility, $1 \mathrm{D}$ helical packing of $\mathrm{Gaq}_{3}$, which has a lower charge-carrier mobility and amorphous rubene, which has very poor charge-carrier mobility.

There are many future experiments that can be carried out to resolve many of these issues. Careful muon studies of the effect of disorder or dopants on eSR may reveal which transport related electron spin relaxation mechanism is relevant. The effect of an electric field, which would act to modify and increase the overall mobility of any free charge carriers, ${ }^{18}$ may also reveal the role of impurity/dopant induced free carriers on eSR. Comparisons of deuterated and undeuterated materials, as well as performing measurements as a function of mass of one of the constituent atoms (e.g., replacing $\mathrm{Ga}$ with $\mathrm{Al}, \mathrm{In}, \mathrm{Tl}$ in $\mathrm{Gaq}_{3}$ ), would help distinguish between the spin-orbit and hyperfine interactions.

It is not entirely clear why two energy regimes to the temperature-dependent eSR are observed, but they may correspond to two groups of vibrations that could not be further resolved. If the interaction is enhanced by the breaking of a 
symmetry, as required for a mechanism involving spin-orbit coupling, then only selected modes may have a relevant symmetry breaking. A possible mechanism could be related to a geometric phase, analogous to the Berry phase, ${ }^{38,39}$ where there would be very few modes that involve molecular motions that sweep out a finite solid angle in one period. This selection rule can explain why there are very few modes contributing, but not why there are exactly two. Alternatively, the mechanism could be related to Bose-Einstein statistics coupled with the need to include vibration emission and absorption processes. ${ }^{40}$ Relaxation processes induced by higher-order spin-vibration couplings, involving simultaneous absorption and emission of vibrational quanta, could also be at the origin of the observed second energy scale.

\section{CONCLUSION}

The eSR was determined with the avoided level crossing (ALC) resonance technique for polycrystalline TIPSpentacene, amorphous rubrene, and polycrystalline $\mathrm{Gaq}_{3}$. The ALC resonance technique is based on $100 \%$ spin-polarized muons, which allows for the spin sensitive measurement of the electron spin relaxation at a molecular level. From the different morphology of the samples as well as the local nature of the used probes, we conclude that the observed electron spin relaxation is an intramolecular process. The relevance of an intramolecular spin relaxation has so far been neglected as it is generally believed that the spin relaxation is governed by the intermolecular motion of the charge carriers. The data presented here therefore indicate that future theoretical models for spin transport in organic materials should also take into account relaxation when the charge carriers are residing on molecules, which could be very significant considering the weak charge-carrier mobilities in these materials.

The obtained eSR at $300 \mathrm{~K}$ of $0.85 \pm 0.02 \mathrm{MHz}$ for $\mathrm{Gaq}_{3}$ and $0.76 \pm 0.03 \mathrm{MHz}$ for TIPS-pentacene are in good agreement with many previously reported values ${ }^{3,7,8}$ for similar materials, but call into question others. ${ }^{6}$ The temperature dependence of the eSR exhibits an Arrhenius-like behavior suggesting a coupling of vibrations to the eSR. Furthermore, the structural difference between the acenes (TIPS-pentacene and rubrene) and the quinolate $\left(\mathrm{Gaq}_{3}\right)$ leads to the conclusion that the observed intramolecular Arrhenius-like spin relaxation may be present in a wide range of organic semiconductors.

\section{ACKNOWLEDGMENTS}

We would like to thank A. Stoykov and R. Scheuermann for the experimental support received at PSI and M. Somerton for the preparation of $\mathrm{Gaq}_{3}$. We would also like to thank E. Roduner, Z. Salman, and R. Scheuermann for valued discussions with regards to the analysis and interpretation of our data. A.J.D. acknowledges financial support from the EPSRC (Grant No. EP/G054568/1) and the Leverhulme Trust. L.S. and C.B. acknowledges financial support from the SNF (Grants No. 200020-119784 and No. 200020-129484) and the NCCR program MaNEP. C.A.S. acknowledges financial support from MaNEP while visiting the University of Fribourg.

\section{APPENDIX}

\section{High transverse field measurements}

The strength of the hyperfine coupling between the muon spin and the electron spin in organic materials can be determined very accurately by performing experiments in a high transverse magnetic field. ${ }^{21,41}$ In a transverse magnetic field, the spin of the free diamagnetic muon precesses at the Larmor frequency. In a high transverse magnetic field (the socalled Paschen-Back limit), for every muonium state, the muon spin precesses at two frequencies, $f_{1}$ and $f_{2}$. They are determined $^{41}$ by the isotropic hyperfine interaction $A$ and the applied magnetic field $B$,

$$
\begin{aligned}
& f_{1}=\frac{A}{2}+\frac{\left(\gamma_{e}-\gamma_{\mu}\right) B}{2}-\frac{A}{2} \sqrt{1+\left(\frac{2 B\left(\gamma_{e}+\gamma_{\mu}\right)}{A}\right)^{2}}, \\
& f_{2}=\frac{A}{2}-\frac{\left(\gamma_{e}-\gamma_{\mu}\right) B}{2}+\frac{A}{2} \sqrt{1+\left(\frac{2 B\left(\gamma_{e}+\gamma_{\mu}\right)}{A}\right)^{2}} .
\end{aligned}
$$

The sum of the two frequencies $f_{1}$ and $f_{2}$ equals the isotropic hyperfine coupling constant $A$. Figure 8(a) shows the Fourier transform of the forward-backward asymmetry measured for TIPS-pentacene at an applied magnetic field of $0.45 \mathrm{~T}$ and at a temperature of $10 \mathrm{~K}$. The external field appears as the strongly pronounced peak at about $60 \mathrm{MHz}$. Symmetrically distanced from this central diamagnetic signal are two groups of satellite peaks at about 20 and $100 \mathrm{MHz}$. From these data, it is clear that the isotropic $\mathrm{HFC}$ is about $80 \mathrm{MHz}$, corresponding to the isotropic hyperfine coupling constants that correspond to the ALC position (see Tables I and III). However, it is also clear that the satellites are rather broad, suggesting several components, but it is not clear how many peak pairs are present. To resolve the lines, we also performed a maximum entropy

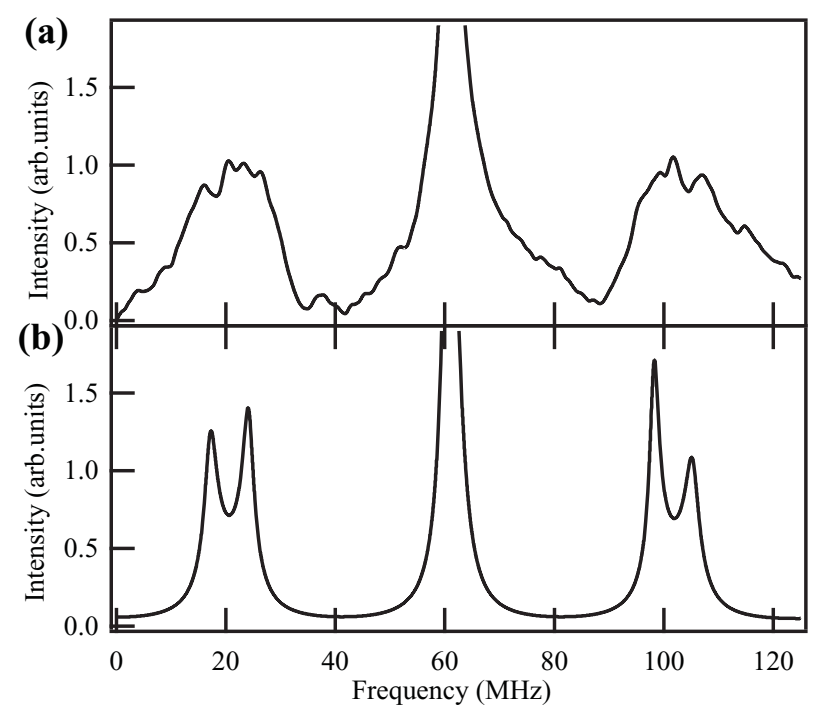

FIG. 8. Transverse field measurements on TIPS-Pentacene, taken at $450 \mathrm{mT}$ and $10 \mathrm{~K}$. In this Fourier spectrum, one can see the truncated peak (external magnetic field) and satellite peaks on either side of the applied field, with the HFCs being consistent to those extracted from the ALC data. The top panel (a) corresponds to a conventional Fourier transform, whereas the bottom panel (b) corresponds to a maximum entropy analysis of the same data. 
analysis. Each satellite peak is clearly split into two discrete lines, as shown in Fig. 8(b), with each pair correspondingly symmetrical about the applied field. The corresponding HFCs are 87.5 and $74.5 \mathrm{MHz}$, which are extremely similar to the values obtained in the ALCs (see Tables I and III). This is a strong indication that there are indeed two different muonium sites on the molecules with very similar HFCs and that the analysis using two independent $\Delta_{1}$ transitions is correct.

\section{Muonium states}

In order to better understand the muoniated radical state and to identify muonium adduct positions, we have performed DFT calculations at the UB3LYP/6-31G level for pentacene and TIPS-pentacene. The obtained isotropic hyperfine coupling constants for the proton and the muon are summarized in Table V. The corresponding assignments are shown in Fig. 9. Unfortunately, the DFT calculations for TIPS-pentacene provide a roughly $40 \%$ higher isotropic HFC than is present in the data, related to the crystallographic packing in our crystalline materials. This makes an unambiguous assignment based on these calculations relatively difficult. However, the two lowest calculated HFCs are very close to one another, similar to the experimental data, suggesting that these two sites could be the ones to follow up experimentally. The potential sites correspond to position 3 on the central pentacene backbone and to position 6 on the side-group triple bond.

We have performed detailed measurements of pentacene [shown in Fig. 10(b)] on a sample that had similar dimensions and mass to the TIPS-pentacene measurements, where position 6 is not present but positon 3 is. We observe an ALC at similar fields to ALC 2 in Fig. 2(b). Interestingly, it is clear that the relative amplitude of the pentacene ALC is significantly smaller than the combination of ALC 1 and 2 observed in TIPS-pentacene [also plotted in Fig. 10(b)]. This suggests that ALC 2 is indeed situated at position 3. Furthermore, the hypothesis of the addition to the triple bond on the side group is supported by a similar HFC in TCNQ, ${ }^{36}$ where the muon addition site has been assigned to the $N$ at the end of the triple bond in the cyano group. ${ }^{33}$
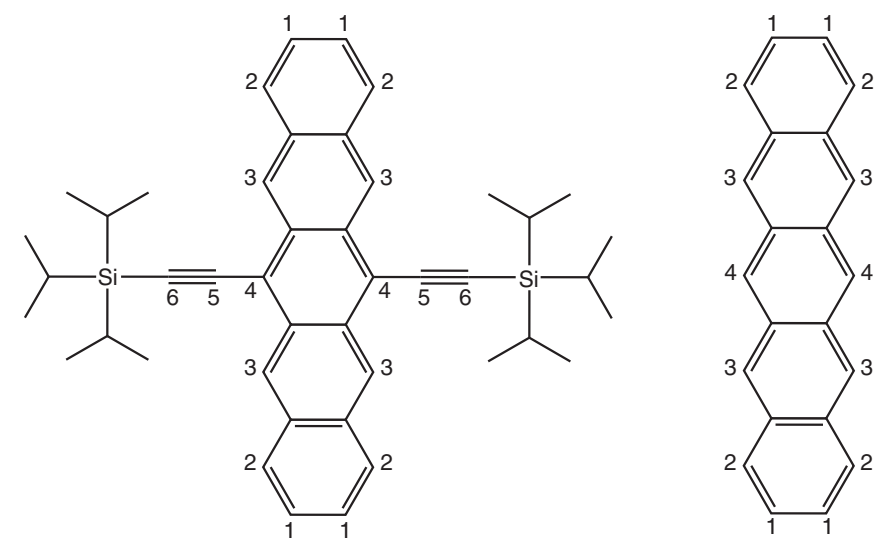

FIG. 9. Position assignment of TIPS-pentacene and pentacene.
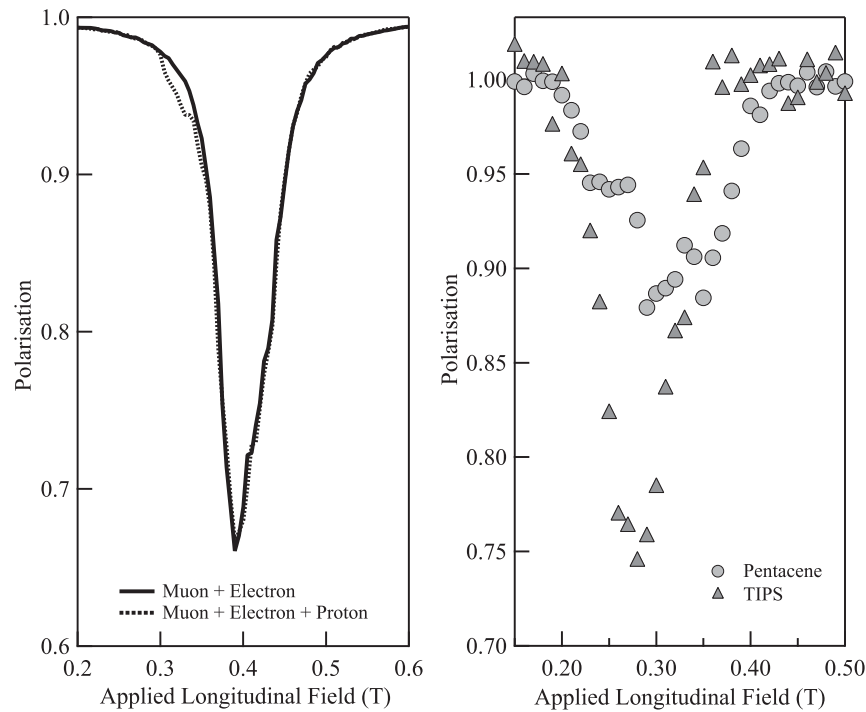

FIG. 10. (a): Calculated ALCs with and without the proton, using $A_{\mu}=109 \mathrm{MHz}, D_{\mu}=10 \mathrm{MHz}, \eta_{\mu}=-5 \mathrm{MHz}$ and $A_{p}=29 \mathrm{MHz}$, $D_{p}=1 \mathrm{MHz}, \eta_{p}=0.3 \mathrm{MHz}$. Isotropic HFCs for the muon and proton were taken from the DFT calculations, whereas the anisotropic HFCs were estimated from the data shown in (b). (b): Comparison of ALC spectra of pentacene and TIPS.

There is therefore good experimental evidence, which is in part backed up by DFT calculations, that suggests that the two muonium sites in TIPS-pentacene are position 3 on the central pentacene backbone and another to position 6 on the side-group triple bond, with positions 3 and 6 corresponding to an isotropic HFC of 86 and $75 \mathrm{MHz}$, respectively. Other ALCs have been detected in TIPS-pentacene at higher magnetic fields, which account for the other possible muonium sites (data not shown). Finally on this subject, we note that in-depth analysis and discussion of the pentacene data and TIPS-pentacene HFCs along with our DFT calculations will be presented elsewhere. ${ }^{42,43}$

Finally, we must be satisfied that the $\Delta_{0}$ ALC resonances are negligibly small. In order to achieve this, we have modeled the spectra using the QUANTUM software ${ }^{31}$ with and without the proton using the parameters in Table V. It is clear from

TABLE V. The muon-electron and muon-proton hyperfine coupling obtained with DFT calculations for several muon sites of pentacene and TIPS/TMS-pentacene. The site number corresponds to the assignment shown in Fig. 9. The HFC could not be determined for $A_{\text {TIPS }}$ site 2 because the calculation did not converge ("n.c."). In some cases it is impossible for the muon to bond to a site (indicated by a dash).

\begin{tabular}{ccccc}
\hline \hline Site & $\begin{array}{c}\text { Pentacene } \\
A_{\mu}(\mathrm{MHz})\end{array}$ & $\begin{array}{c}\text { Pentacene } \\
A_{p}(\mathrm{MHz})\end{array}$ & $\begin{array}{c}\text { TIPS } \\
A_{\mu}(\mathrm{MHz})\end{array}$ & $\begin{array}{c}\text { TIPS } \\
A_{p}(\mathrm{MHz})\end{array}$ \\
\hline 1 & 279.49 & 64.21 & 253.43 & 63.70 \\
2 & 255.47 & 70.24 & n.c. & n.c. \\
3 & 121.60 & 30.56 & 114.40 & 28.75 \\
4 & 98.07 & 24.65 & - & - \\
5 & - & - & 662.32 & - \\
6 & - & - & 109.13 & - \\
\hline \hline
\end{tabular}


Fig. 10(a) that the difference between the two spectra is marginal, offering support to the conclusion that we have two individual $\Delta_{1}$ transitions resulting from two addition sites. The conclusion that $\Delta_{0}$ ALC resonances are small in solids is also supported experimentally, where ALC spectra were observed in benzene in ZSM5 silicate as a function of temperature. ${ }^{44}$ At the lowest temperatures, where the benzene molecules were essentially static, the $\Delta_{0}$ is very small. As the temperature is increased, and rotational dynamics become important, the $\Delta_{0}$ transition gains significantly in amplitude.
${ }^{1}$ M. Berggren, D. Nilsson, and N. D. Robinson, Nat. Mater. 6, 3 (2007).

${ }^{2}$ G. Szulczewski, S. Sanvito, and M. Coey, Nat. Mater. 8, 693 (2009). ${ }^{3}$ V. A. Dediu, L. E. Hueso, I. Bergenti, and C. Taliani, Nat. Mater. 8, 707 (2009).

${ }^{4}$ A. J. Drew, J. Hoppler, L. Schulz, F. L. Pratt, P. Desai, P. Shakya, T. Kreouzis, W. P. Gillin, A. Suter, N. A. Morley, V. K. Malik, A. Dubroka, K. W. Kim, H. Bouyanfif, F. Bourqui, C. Bernhard, R. Scheuermann, G. J. Nieuwenhuys, T. Prokscha, and E. Morenzoni, Nat. Mater. 8, 109 (2009).

${ }^{5}$ M. Cinchetti, K. Heimer, J.-P. Westenberg, O. Andreyev, M. Bauer, S. Lach, C. Ziegler, Y. Gao, and M. Aeschlimann, Nat. Mater. 8, 115 (2009).

${ }^{6}$ S. Pramanik, C.-G. Stefanita, S. Patibandla, S. Bandyopadhyay, K. Garre, N. Harth, and M. Cahay, Nat. Nano. 2, 216 (2007).

${ }^{7}$ J. Rybicki and M. Wohlgenannt, Phys. Rev. B 79, 153202 (2009).

${ }^{8}$ V. Dediu, M. Murgia, F. C. Matacotta, C. Taliani, and S. Barbanera, Solid State Commun. 122, 181 (2002).

${ }^{9}$ R. J. Elliott, Phys. Rev. 96, 266 (1954)

${ }^{10}$ M. I. D'yakonov and V. Perel, Sov. Phys. JETP 33, 1053 (1971).

${ }^{11}$ P. A. Bobbert, W. Wagemans, F. W. A. van Oost, B. Koopmans, and M. Wohlgenannt, Phys. Rev. Lett. 102, 156604 (2009).

${ }^{12}$ H. Park, D.-S. Shin, H.-S. Yu, and H.-B. Chae, Appl. Phys. Lett. 90, 202103 (2007).

${ }^{13}$ J. E. Anthony, Chem. Rev. 106, 5028 (2006).

${ }^{14}$ J. E. Anthony, D. L. Eaton, and S. R. Parkin, Org. Lett. 4, 15 (2002).

${ }^{15}$ I. Hernandez and W. P. Gillin, J. Phys. Chem. B 113, 14079 (2009).

${ }^{16}$ S. K. Park, T. N. Jackson, J. E. Anthony, and D. A. Mourey, Appl. Phys. Lett. 91, 063514 (2007).

${ }^{17}$ D. H. Kim, D. Y. Lee, H. S. Lee, W. H. Lee, Y. H. Kim, J. I. Han, and K. Cho, Adv. Mater. 19, 678 (2007).

${ }^{18}$ A. J. Drew, F. L. Pratt, J. Hoppler, L. Schulz, V. Malik-Kumar, N. A. Morley, P. Desai, P. Shakya, T. Kreouzis, W. P. Gillin, K. W. Kim, A. Dubroka, and R. Scheuermann, Phys. Rev. Lett. 100, 116601 (2008).

${ }^{19}$ E. Roduner, The Positive Muon as a Probe in Free Radical Chemistry, Lecture Notes in Chemistry. No. 49 (Springer, Berlin, 1988).

${ }^{20}$ F. L. Pratt, J. Phys. Cond. Mat. 16, S4779 (2004).

${ }^{21}$ B. Patterson, Rev. Mod. Phys. 60, 69 (1988).
${ }^{22}$ J. E. Anthony, J. S. Brooks, D. L. Eaton, and S. R. Parkin, J. Am. Chem. Soc. 123, 9482 (2001).

${ }^{23}$ O. D. Jurchescu, A. Meetsma, and T. T. M. Palstra, Acta Crystallogr., Sect. B 62, 330 (2006).

${ }^{24}$ M. Brinkmann, G. Gadret, M. Muccini, C. Taliani, N. Masciocchi, and A. Sironi, J. Am. Chem. Soc. 122, 5147 (2000).

${ }^{25}$ S. R. Keitzman, B. Hitti, R. L. Lichti, T. L. Estle, and K. H. Chow, Chem. Phys. 192, 189 (1995).

${ }^{26}$ E. Roduner, Hyperfine Interact. 65, 857 (1990).

${ }^{27}$ M. Heming, E. Roduner, I. D. Reid, P. W. F. Louwrier, J. W. Schneider, H. Keller, W. Odermatt, B. D. Patterson, H. Simmler, B. Pumpin, and I. M. Savic, Chem. Phys. 129, 335 (1989).

${ }^{28} \mathrm{M}$. Heming, E. Roduner, and B. D. Patterson, Hyperfine Interact. 32, 727 (1986)

${ }^{29}$ S. Blundell, Chem. Rev. 104, 5717 (2004).

${ }^{30}$ F. L. Pratt, Physica B 289, 710 (2000).

${ }^{31}$ J. S. Lord, Physica B 374-375, 472 (2006).

${ }^{32}$ E. Roduner, K. Prassides, R. M. Macrae, I. M. Thomas, C. Niedermayer, U. Binninger, C. Bernhard, A. Hofer, and I. D. Reid, Chem. Phys. 192, 231 (1995).

${ }^{33}$ R. M. Macrae, Magn. Reson. Chem. 38, S33 (2000).

${ }^{34}$ I. McKenzie, J. Phys. Chem. A 114, 12759 (2010).

${ }^{35}$ P. Desai, P. Shakya, T. Kreouzis, W. P. Gillin, N. A. Morley, and M. R. J. Gibbs, Phys. Rev. B 75, 094423 (2007).

${ }^{36}$ F. L. Pratt, S. J. Blundell, T. Jestadt, B. W. Lovett, R. M. Macrae, and W. Hayes, Magn. Reson. Chem. 38, S27 (2000).

${ }^{37}$ N. J. Rolfe, M. Heeney, P. B. Wyatt, A. J. Drew, T. Kreouzis, and W. P. Gillin, Phys. Rev. B 80, 241201 (2009).

${ }^{38}$ P. San-Jose, G. Zarand, A. Shnirman, and G. Schön, Phys. Rev. Lett. 97, 076803 (2006).

${ }^{39}$ P. San-Jose, B. Scharfenberger, G. Schön, A. Shnirman, and G. Zarand, Phys. Rev. B 77, 045305 (2008).

${ }^{40}$ S. Guha, J. D. Rice, Y. T. Yau, C. M. Martin, M. Chandrasekhar, H. R. Chandrasekhar, R. Guentner, P. S. de Freitas, and U. Scherf, Phys. Rev. B 67, 125204 (2003).

${ }^{41}$ R. F. Kiefl, E. Holzschuh, H. Keller, W. Kündig, P. F. Meier, B. D. Patterson, J. W. Schneider, K. W. Blazey, S. L. Rudaz, and A. B. Denison, Phys. Rev. Lett. 53, 90 (1984).

${ }^{42} \mathrm{M}$. Willis et al. (unpublished).

${ }^{43}$ L. Schulz et al. (unpublished).

${ }^{44}$ E. Roduner, M. Stolmar, H. Dilger, and I. D. Reid, J. Phys. Chem. A 102, 7591 (1998). 